\title{
CXCR2-transduced Autologous Tumor Infiltrating Lymphocytes
}

National Cancer Institute

\section{Source}

National Cancer Institute. CXCR2-transduced Autologous Tumor Infiltrating

Lymphocytes. NCI Thesaurus. Code C120001.

A preparation of autologous tumor infiltrating lymphocytes (TILS) that are transduced, ex vivo, with a retroviral vector encoding a gene for CXC chemokine receptor 2 (CXCR2), with potential antineoplastic activity. Upon administration of the CXCR2-transduced autologous TILs, the CXCR2-expressing T-cells selectively migrate toward tumor cells expressing CXCR2 ligands, which leads to tumor cell killing. CXCR2 expression allows for optimal TIL migration towards tumor cells and enhances the TILs anti-tumor activity. This leads to a reduction in both tumor cell proliferation and survival. CXCR2, a transmembrane protein also known as IL-8 receptor B (IL-8RB), plays a key role in inflammation and cancer progression. Certain CXCR2 ligands, such as CXCL1 and CXCL8 (IL-8), are expressed by tumor cells. 